\title{
Novel Conjugated Polymer Containing Anthracene Backbone: Competitive Addition Polymerization of Dithiol Monomers to Diethynyl Monomers and Relative Reactivity
}

\author{
Eiichi KobAYASHI, ${ }^{*}$ Jian JiAng, Sadahito Aoshima, \\ and Junji FURUKAWA \\ Department of Industrial Chemistry, Faculty of Science and \\ Technology, Science University of Tokyo, \\ Noda, Chiba 278, Japan
}

(Received September 28, 1989)

\begin{abstract}
Competitive addition polymerizations of 9,10-anthracenedithiol, 4,4'-biphenyldithiol, and 1,4-benzenedithiol to 9,10-diethynylanthracene, 4,4'-diethynylbiphenyl, and 1,4diethynylbenzene were carried out in THF at $50^{\circ} \mathrm{C}$ by UV irradiation under nitrogen atmosphere, and the relative reactivities of the monomers are discussed on the basis of composition analysis for the obtained polymers. To the dithiol monomers, the relative reactivities of diethynyl monomers are in the order of 9,10-diethynylanthracene $>4,4^{\prime}$-diethynylbiphenyl $>1,4$-diethynylbenzene. The relative reactivities of dithiol monomers to the diethynyl monomers are in the order of 9,10 anthracenedithiol $>4,4^{\prime}$-biphenyldithiol $>1$,4-benzenedithiol. These results indicate that the more the monomer conjugation, the higher is the relative reactivity. The high reactivities of anthracene derivatives would be caused from stabilization of the intermediate carbon radical and thio radical by its high conjugation system.
\end{abstract}

KEY WORDS Conjugated Polymer / 9,10-Diethynylanthracene / 9,10Anthracenedithiol / 4,4'-Diethynylbiphenyl / 4,4'-Biphenyldithiol / 1,4Diethynylbenzene / 1,4-Benzenedithiol / Competitive Addition Polymerization / Relative Reactivity /

The addition reaction of mercaptanes onto the carbon-carbon triple bond in a radical mechanism yields anti-Markownikoff products. $^{1}$ UV induced addition polymerization of 1,4-benzenedithiol onto 1,4-diethynylbenzene in toluene solution ${ }^{2}$ and in solid state $^{3}$ gave an insoluble polymer with a number average molecular weight $\left(\bar{M}_{n}\right)$ of $3000-7000$. Interestingly, the polymer obtained in the latter case was the crystalline polymer, and the crystallinity and microstructure (cis-trans contents of vinylene) can be changed reversibly by irradiation of the light with characteristic wavelengths. ${ }^{4}$ The biphenyl derivatives, 4,4'diethynylbiphenyl and 4,4'-biphenyldithiol, exhibited the same polymerization behavior. A crystalline polymer was also obtained in the solid state polymerization by UV irradiation, ${ }^{5}$ and the $\mathrm{X}$-ray diffraction pattern of the crystal may be changed by the thermal treatment. Recently, the authors synthesized new monomers, 9,10-diethynylanthracene and 9,10-anthracenedithiol, and carried out an addition polymerization in THF to obtain the corresponding conjugated polymers containing anthracene rings in the polymer backbone. ${ }^{6.7}$ The above polymers have relatively lower decomposition temperature $\left(350^{\circ} \mathrm{C}\right)$, because of the unstability of anthracene ring against oxidation than that of benzene

* To whom all correspondence should be addressed. 
$\left(500^{\circ} \mathrm{C}\right)$. However, the polymers have higher molecular weights, about 70000 to 80000 , than that of the polymer composed of benzene or biphenyl derivatives. Reactivities among these monomers may control the molecular weights of the polymers.

Recently, Nuyken and Siebzehnrübl prepared telechelic polymers having a thiol end group with the method of unstoichiometric ratio of monomers in the feed in the polymerization of 1,3-benzenedithiol with 1,5-hexadiyne and 1,6-heptadiyne. 8,9 These polymers synthesized by the above method have a special feature of dissolving in conventional organic solvents such as benzene, toluene, chloroform, and THF.

In this paper, the authors evaluated relative reactivities among these anthracene, biphenyl and benzene derivatives by competitive polymerization and discuss the reactivities of monomers and the polymerization mechanism.

\section{EXPERIMENTAL}

\section{Monomers}

The monomers used in the polymerization were synthesized and purified according to the references; they are 1,4-diethynylbenzene (DEB) and 1,4-benzenedithiol (BDT), ${ }^{3} 4,4^{\prime}$ diethynylbiphenyl (DEBP) and 4,4'-biphenyldithiol (BPDT) ${ }^{5}$ 9,10-diethynylanthracene (DEA) and 9,10-anthracenedithiol (ADT). ${ }^{6,7}$

\section{Competitive Polymerization}

The competitive polymerization of ADT and BDT to DEA was carried out in THF solution at $50^{\circ} \mathrm{C}$ by UV irradiation, using high pressure mercury lamp (450W, Ushio Co.) under nitrogen atmosphere. For instance, the monomers were charged so that the molar ratio of DEA, ADT, and $\mathrm{BDT}$ was $1: 0.5: 0.5$. After the polymerization was quenched by freezing in liquid nitrogen, the precipitated insoluble polymer was recovered by centrifugation, washed with THF several times and dried in vacuo at room temperature overnight. On the other hand, the soluble polymer in the residual solution was poured into methanol, and the precipitated polymer was also collected as a soluble fraction in the same manner above.

\section{Characterization}

The following instruments and methods were used for the characterization of the monomers and obtained polymers: ${ }^{1} \mathrm{H}$ NMR, JEOL PMX-60si; ${ }^{13} \mathrm{C}$ NMR, JNM FX-90Q; IR, Hitachi 260-50; UV, Shimadzu UV-260; Diffuse reflectance spectra, Hitachi UV-300. The monomer unit compositions in the polymers were obtained by calculation of $S$ content in the polymers, determined by the Schoeniger's method. ${ }^{2}$ The number average molecular weights of the polymers were measured by the method for determining end groups, the so called copper acetylide method. $^{2}$

The electroconductivity of the polymers was measured by the following two-terminal method with an Electro Multi Meter (Takeda Riken Co.). $\mathrm{I}_{2}$ doping was carried out by exposing the polymer powder to iodine vapor at $40^{\circ} \mathrm{C}$ under reduced pressure, $0.5 \mathrm{mmHg}$, and then the doped polymer was pressed to a pellet to determine electroconductivity.

\section{RESULTS AND DISCUSSION}

\section{Polymerization}

The addition polymerizations of aromatic dithiol derivatives to diethynyl derivatives easily took place and gave high molecular weight polymers except a pair of the biphenyl derivatives (see Table I). THF is a more suitable solvent than toluene for obtaining high molecular weight polymer. The yields of the insoluble polymers decreased with the size of the monomers. Perhaps, the poor solubility of anthracene derivatives in reaction solution may affect the yields of the polymers. Both the calculated and observed sulfur contents of the 
Table I. Addition polymerization of equimolar of dithiol to diethynyl derivatives in $\mathrm{THF}$ at $50^{\circ} \mathrm{C}$ for $120 \mathrm{~min}$ by UV irradiation under nitrogen atmosphere ${ }^{\mathrm{a}}$

\begin{tabular}{|c|c|c|c|c|c|c|c|c|}
\hline \multirow{2}{*}{ No. } & \multicolumn{2}{|c|}{ Monomers } & \multicolumn{2}{|c|}{ Yield $/ \%$} & \multicolumn{2}{|c|}{$\mathrm{Swt} \% \mathrm{~b}$} & \multicolumn{2}{|c|}{$\bar{M}_{n} \times 10^{-3 \mathrm{c}}$} \\
\hline & Diethynyl & Dithiol & Insol. & Sol. & Calcd. & Found & Insol. & Sol. \\
\hline 1 & DEB & BDT & 71 & 22 & 23.9 & 23.7 & $49^{d}$ & 6 \\
\hline 2 & DEBP & BPDT & 59 & Trace & 15.2 & 13.9 & $3^{e}$ & - \\
\hline 3 & DEA & BDT & 55 & 21 & 17.4 & 17.2 & 89 & 7 \\
\hline 4 & DEA & ADT & 24 & 42 & 13.7 & 13.9 & 50 & 5 \\
\hline
\end{tabular}

a $[$ diethynyl $]=[$ dithiol $]=8.5 \times 10^{-2} \mathrm{moll}^{-1}$.

b $\mathrm{Swt} \%$ of insoluble polymer determined by Schoeniger's method.

c Copper acetylide method.

d 7500 polymerized in toluene at $-20^{\circ} \mathrm{C}$.

e $[D E B P]=[B P D T]=5 \times 10^{-2} \mathrm{moll}^{-1}$ in toluene at $34^{\circ} \mathrm{C}$ for $10 \mathrm{~min} .^{5}$

Table II. Characterization of the insoluble polymers ${ }^{a}$

\begin{tabular}{|c|c|c|c|c|c|c|}
\hline \multirow{2}{*}{ No. } & \multirow{2}{*}{ Polymer } & \multirow{2}{*}{$\bar{M}_{n} \times 10^{-3 \mathrm{~b}}$} & \multirow{2}{*}{ Color } & \multirow{2}{*}{$\frac{\lambda_{\max }{ }^{c}}{n m}$} & \multicolumn{2}{|c|}{ Conductivity $/ \mathrm{S} \mathrm{cm}^{-1 \mathrm{~d}}$} \\
\hline & & & & & Undoped & Doped \\
\hline 1 & -DEB-BDT- & 49 & Yellowish & 380 & $2.0 \times 10^{-13}$ & $\begin{array}{l}1.3 \times 10^{-8} \\
(0.20)\end{array}$ \\
\hline 2 & -DEBP-BPDT- & 3 & Yellowish & 380 & $1.0 \times 10^{-7}$ & $\begin{array}{c}1.7 \times 10^{-5} \\
(0.32)\end{array}$ \\
\hline 3 & -DEA-BDT- & 89 & Brownish & 428 & $2.0 \times 10^{-13}$ & $\begin{array}{c}1.6 \times 10^{-6} \\
(0.89)\end{array}$ \\
\hline 4 & -DEA-ADT- & 50 & Brown & 580 & $9.6 \times 10^{-12}$ & $\begin{array}{c}2.9 \times 10^{-6} \\
(0.53)\end{array}$ \\
\hline
\end{tabular}

a For conditions of polymerization, see Table I.

b Copper acetylide method.

c Diffuse reflectance spectra.

d Two-terminal method; ( ) is the amount of $I_{2}$ mole per repeating unit.

insoluble polymers were almost the same except for the DEBP-BPDT, which illustrated that the obtained polymers were composed of diethynyl and the dithiol monomer units in the same molar ratios.

The characterization of the insoluble polymers is shown in Table II. The obtained insoluble polymers were in the form of powders, and changed from yellowish to brown, when the phenylene unit in the polymer backbone was substituted with the anthracene ring. The maximum absorption wavelengths of the insoluble polymers also showed the same tendency and indicated that the anthracene ring in the polymers could make the conjugation system wider along the polymer backbone. The electroconductivities of the undoped polymers were in the order of insulator, about $10^{-13} \mathrm{~S} \mathrm{~cm}^{-1}$, but increased up to $10^{-6} \mathrm{~S} \mathrm{~cm}^{-1}$ with $\mathrm{I}_{2}$ doping. $\mathrm{I}_{2}$ doping could not enhance the conductivity much more. Conductivity could be improved considerably if more suitable dopants are chosen.

\section{Competitive Addition Polymerization}

The polymers mentioned above are known to be conjugated polymers containing sulfur and aromatic rings in the polymer backbone. 


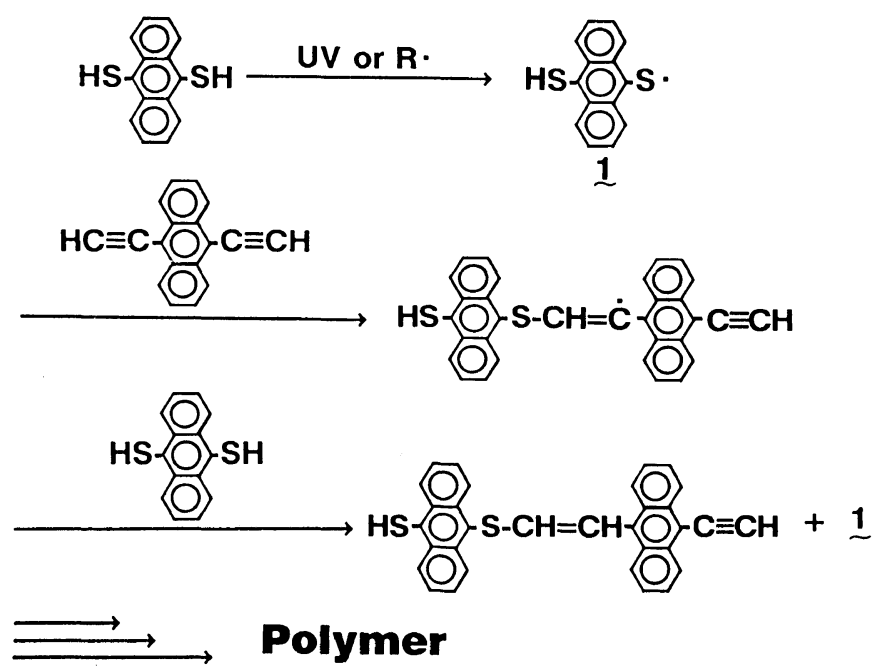

Scheme 1. The mechanism of polymerization.

However, there have been few studies about the polymerization mechanism, properties $^{2-7}$ and monomer reactivities, because the obtained polymers do not dissolve in any organic solvents.

The polymerization of DEA with ADT may take place by the mechanism shown in Scheme 1. By irradiation of UV light or existence of a radical initiator, ADT would be broken to generate an anthracenethio radical, which attacks the carbon-carbon triple bond of DEA to give a relatively stable intermediate carbon radical. The carbon radical could abstract a hydrogen from ADT to form a dimer and another anthracenethio radical. In the same way, the newly formed oligomers have thiol and ethynyl groups, so that they also take part in the radical addition polymerization. A high polymer could be obtained with the repetition of the above steps.

The addition reaction of anthracenethio radical to the carbon-carbon triple bond may be the rate determining step of the polymerization. This idea was supported by a model addition reaction work done by this laboratory, ${ }^{10}$ in which the addition reaction of thiophenol to ethynylbenzene was studied and the obtained results showed the rate of addi- tion reaction to depend on the concentration of ethynylbenzene but not on thiophenol.

In order to elucidate the polymerization mechanism and relative reactivities among the benzene, biphenyl and anthracene derivatives, competitive polymerizations were carried out in THF and the behavior is also discussed.

Table III shows an example of the competitive polymerization of ADT to DEA and DEB in THF at $50^{\circ} \mathrm{C}$ by UV irradiation under nitrogen atmosphere. From the observed $\mathrm{S}$ content of the polymer, it was known that in the polymer, the ADT-DEA unit was $76 \%$ and the ADT-DEB unit $24 \%$. This result clearly indicates that the relative reactivity of DEA to ADT is about 3 times higher than that of DEB.

In the same manner, the relative reactivity of ADT to DEBP and DEB was also examined and the order of reactivities of DEA, DEBP, and DEB to the ADT was determined to be $1.0,0.6$, and 0.3 , respectively.

The relative reactivities of these diethynyl monomers to the dithiol monomers are summarized in Table IV. It may be concluded that in the dithiol monomers, the more the monomer conjugation, the higher is the reactivity of the diethynyl monomer.

The relative reactivities of the dithiol mono- 
Table III. Relative reactivity of ADT to DEA and $\mathrm{DEB}$ in THF at $50^{\circ} \mathrm{C}$ by $\mathrm{UV}$ irradiation under nitrogen atmosphere

\begin{tabular}{|c|c|}
\hline Monomer composition & Polymer composition \\
\hline$\times 10^{-2} \mathrm{moll}^{-1}$ & $\mathrm{~mol}_{\mathrm{o}}^{\mathrm{b}}$ \\
\hline $\begin{aligned} {[\mathrm{DEA}]=} & 2.1 \\
& +[\mathrm{ADT}]=4.2\end{aligned}$ & $\begin{array}{c}- \text { DEA-ADT- } \\
76\end{array}$ \\
\hline$[\mathrm{DEB}]=2.1$ & $\begin{array}{c}\text {-DEB-ADT- } \\
24\end{array}$ \\
\hline
\end{tabular}

a Polymer yield $=25 \% ; \bar{M}_{n}=7.0 \times 10^{4}$.

b Calculated based on $\mathrm{S} w \mathrm{t} \%$ of polymer.

Table IV. Relative reactivities of diethynyl monomers to dithiol monomers in THF at $50^{\circ} \mathrm{C}$ by UV irradiation under nitrogen atmosphere

\begin{tabular}{lrcc}
\hline & \multicolumn{3}{c}{ Diethynyl } \\
\cline { 2 - 4 } Dithiol & DEA & DEBP & DEB \\
\hline ADT & 1.0 & 0.6 & 0.3 \\
BPDT & 3.0 & 1.0 & 0.2 \\
BDT & 11.5 & 6.7 & 1.0 \\
\hline
\end{tabular}

[diethynyl monomer 1] =[diethynyl monomer 2] = $1 / 2$ [dithiol monomer] $=2.1 \times 10^{-2} \mathrm{moll}^{-1}$. Yield was less than $25 \%$.

mers to the diethynyl monomers are summarized in Table $\mathrm{V}$, in which the results also indicate that to diethynyl monomers, the highly conjugated monomer (ADT) has the highest reactivity, then the second is BPDT followed by BDT, except in the case of ADT to DEB. The reason for the high reactivity of the highly conjugated monomer may be that the resonance stability of newly formed thio radical of highly conjugated dithiol monomers is higher than that of the low conjugated dithiol monomers. Simple quantum chemical calculations indicated that the SOMO of the highly conjugated thio radical could easily interact with the HOMO of ethynyl group. Thus, the higher the conjugation of thiol monomer, the easier is the addition of the thio radical to
Table V. Relative reactivities of dithiol monomers to diethynyl monomers in THF at $50^{\circ} \mathrm{C}$ by UV irradiation under nitrogen atmosphere

\begin{tabular}{lccc}
\hline \multirow{2}{*}{ Diethynyl } & \multicolumn{3}{c}{ Dithiol } \\
\cline { 2 - 4 } & ADT & BPDT & BDT \\
\hline DEA & 1.0 & 0.4 & 0.2 \\
DEBP & 3.0 & 1.0 & 0.4 \\
DEB & $(2.2)$ & 2.8 & 1.0 \\
\hline
\end{tabular}

[dithiol monomer 1] $=[$ dithiol monomer 2$]=1 / 2[\mathrm{di}$ ethynyl monomer $]=2.1 \times 10^{-2} \mathrm{moll}^{-1}$. Yield was less than $25 \%$.

Table VI. ${ }^{13} \mathrm{C}$ NMR chemical shifts of diethynyl monomers in $\mathrm{CDCl}_{3}$, TMS as the internal standard

\begin{tabular}{|c|c|c|c|}
\hline \multirow{2}{*}{$\mathrm{HC} \equiv \mathrm{C}-\mathrm{Ar}-\stackrel{\alpha}{\mathrm{C}} \equiv \stackrel{\beta}{\mathrm{C}} \mathrm{H}$} & \multicolumn{2}{|c|}{ Chemical shifts/ppm } & \multirow{2}{*}{$\frac{\Delta}{C_{\alpha}-C_{\beta}}$} \\
\hline & $C_{\alpha}$ & $C_{\beta}$ & \\
\hline Anthracene & 89.8 & 80.2 & 9.6 \\
\hline Biphenyl & 83.4 & 78.1 & 5.3 \\
\hline Benzene & $\begin{array}{c}83.0 \\
(82.9)\end{array}$ & $\begin{array}{c}79.1 \\
(80.6)^{\mathrm{a}}\end{array}$ & 3.9 \\
\hline
\end{tabular}

a D. M. White and G. C. Levy, Macromolecules, 5, 527 (1972).

ethynyl group, so that the combination of DEA and ADT has the highest reactivity. Detailed quantum chemical calculations on these addition reactions and kinetic study of the substituted phenylacetylene and thiophenol are now in progress.

Table VI shows the ${ }^{13} \mathrm{C}$ NMR data to compare the relative electron density of the ethynyl groups of diethynyl monomers. From the ${ }^{13} \mathrm{C}$ NMR chemical shift, the values $\Delta=$ $C_{\alpha}-C_{\beta}$ are representative of the difference of the electron density in the $\alpha$ and $\beta$ carbons and indicate the magnitude of the polarization of the acetylenic bond induced by the $-\mathrm{R}$ effect of the aromatic group. The values of $\Delta$ are in good agreement with the order of relative reactivities of diethynyl monomers obtained from the results of competitive polymerization. It is expected from the above results 


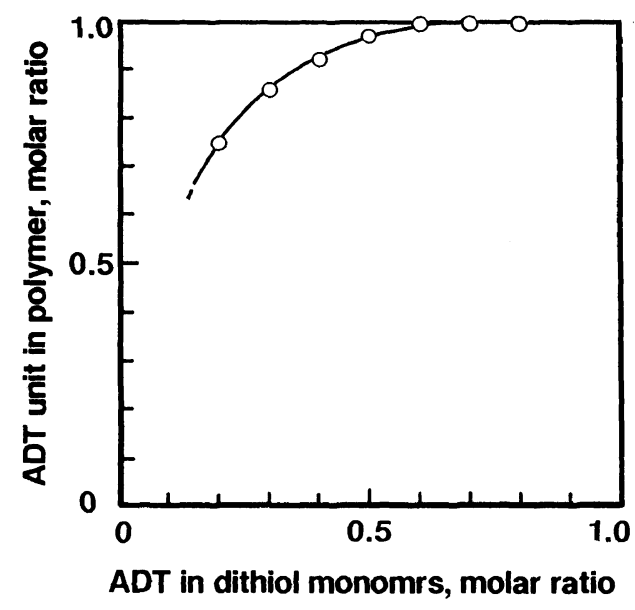

Figure 1. Monomer $v s$. polymer composition curve polymerized in THF at $50^{\circ} \mathrm{C}$ for $1 \mathrm{~min}$ by UV irradiation under nitrogen atmosphere $([\mathrm{ADT}]+[\mathrm{BDT}]=[\mathrm{DEA}]=$ $2.3 \times 10^{-2} \mathrm{moll}^{-1}$, yield was less than $32 \%$ ).

that the rate determining step of the polymerization is the step of the thio radical attacking the ethynyl end group to form a stable vinylene radical. Its stability would be controlld by the magnitude of the conjugation of the adjacent aromatic group. Consequently, the relative reactivities decreased in the order of anthracene $>$ biphenyl $>$ benzene.

\section{Effect of the Monomer Feed Ratio}

The monomer feed ratio can affect the composition and molecular weight of the obtained polymers. In the competitive polymerization of ADT and BDT to DEA, the fraction of the ADT unit in the obtained polymer is always higher than that of BDT, even though the molar ratio of $\mathrm{BDT}$ in the monomer feed changed from 0.2 to 0.8 as shown in Figure 1. This behavior is evidence that the reactivity of ADT to DEA is higher than that of BDT in wide range of monomer feed.

Figure 2 shows the effect of the monomer feed ratio on the yield and molecular weight of the polymers. The yield of the polymer was less than $32 \%$, and the average degree of polymerization was about $4-9$. It is clear that both the yield and the molecular weight increase with

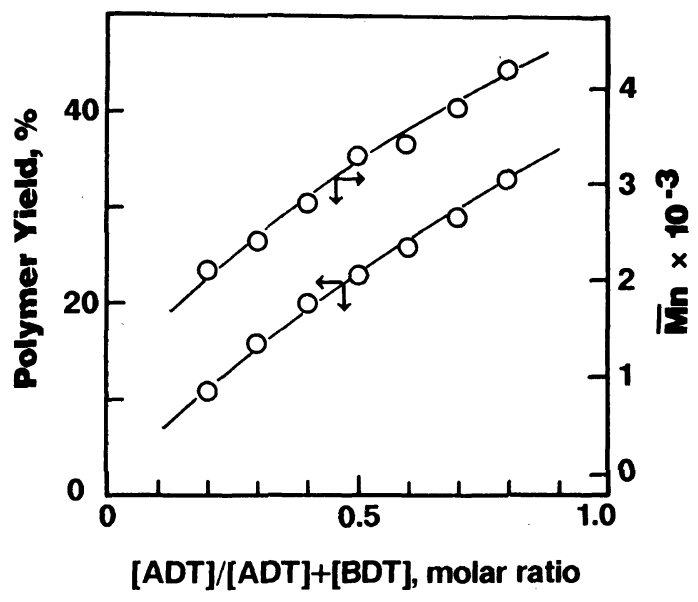

Figure 2. Effect of monomer feed ratio on the yield and $\bar{M}_{n}$ of polymer polymerized in THF at $50^{\circ} \mathrm{C}$ for $1 \mathrm{~min}$ by UV irradiation under nitrogen atmosphere $\left([\mathrm{ADT}]+[\mathrm{BDT}]=[\mathrm{DEA}]=2.3 \times 10^{-2} \mathrm{moll}^{-1}\right.$, yield was less than $32 \%$ ).

the molar ratio of ADT in monomer feed. The high reactivity of the ADT caused the high yield and high molecular weight of the polymers.

\section{CONCLUSIONS}

The relative reactivities in the addition polymerization of ADT, BPDT, and BDT to DEA, DEBP, and DEB were studied by competitive polymerization in $\mathrm{THF}$ at $50^{\circ} \mathrm{C}$ by UV irradiation under nitrogen atmosphere. To the dithiol monomers the relative reactivity of diethynyl monomers is in the order of $\mathrm{DEA}>$ DEBP $>$ DEB. The relative reactivity of the dithiol monomers to the diethynyl monomers is in the order of ADT $>$ BPDT $>$ BDT. This order is not altered by changing the molar ratio of the monomer feed. The highest reactivity of anthracene monomers among these monomers would be caused from resonance stabilization of the intermediate carbon radicals and thio radicals caused by its high conjugation system. 


\section{REFERENCES}

1. J. March, "Advanced Organic Chemistry," Wiley and Sons, New York, N.Y., 1985, p 678.

2. E. Kobayashi, T. Ohashi, and J. Furukawa, Makromol. Chem., 187, 2525 (1986).

3. E. Kobayashi, T. Ohashi, and J. Furukawa, J. Polym. Sci., Polym. Chem. Ed., 25, 2077 (1987).

4. E. Kobayashi, T. Ohashi, T. Yagi, and J. Furukawa, Report of Japan Synthetic Fiber Institute, 44, 95 (1987).

5. E. Kobayashi, T. Kaneda, T. Ohashi, and J.
Furukawa, Polym. Prepr. Jpn., 37, 264 (1988).

6. E. Kobayashi, J. Jiang, and J. Furukawa, to be published in Polym. J.

7. E. Kobayashi, J. Jiang, H. Ohta, and J. Furukawa, to be published in J. Polym. Sci., Polym. Chem. Ed.

8. O. Nuyken and F. Siebzehnrübl, Polym. Bull., 19, 371 (1988).

9. O. Nuyken and F. Siebzehnrübl, Makromol. Chem., 189, 541 (1988).

10. E. Kobayashi, T. Obata, S. Aoshima, and J. Furukawa, Polym. Prepr. Jpn., 38, 332 (1989). 